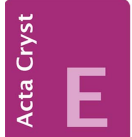
COMMUNICATIONS

ISSN 2056-9890

\section{Crystal structure of 2,5-dimethyl-3-(2- methylphenylsulfinyl)-1-benzofuran}

\author{
Hong Dae Choi ${ }^{\mathrm{a}}$ and Uk Lee ${ }^{\mathrm{b} *}$
}

a Department of Chemistry, Dongeui University, San 24 Kaya-dong, Busanjin-gu, Busan 614-714, Republic of Korea, and 'bepartment of Chemistry, Pukyong National University, 599-1 Daeyeon 3-dong, Nam-gu, Busan 608-737, Republic of Korea. ${ }^{*}$ Correspondence e-mail: uklee@pknu.ac.kr

Received 30 June 2015; accepted 2 July 2015

Edited by V. V. Chernyshev, Moscow State University, Russia

In the title compound, $\mathrm{C}_{17} \mathrm{H}_{16} \mathrm{O}_{2} \mathrm{~S}$, the dihedral angle between the benzofuran ring system [r.m.s. deviation $=0.009$ (1) $\AA$ ] and the 2-methylphenyl ring is $86.72(4)^{\circ}$. In the crystal, weak C$\mathrm{H} \cdots \mathrm{O}$ hydrogen bonds link the molecules into columns along the $b$-axis direction.

Keywords: crystal structure; benzofuran; 2-methylphenyl; C- $\mathrm{H} \cdots \mathrm{O}$ hydrogen bonds.

CCDC reference: 1410058

\section{Related literature}

For the pharmacological properties of benzofuran compounds, see: Aslam et al. (2009); Galal et al. (2009); Howlett et al. (1999); Wahab Khan et al. (2005); Ono et al. (2002). For natural products with a benzofuran ring, see: Akgul \& Anil (2003); Soekamto et al. (2003). For a related structure, see: Choi et al. (2012). For further synthetic details, see: Choi et al. (1999).

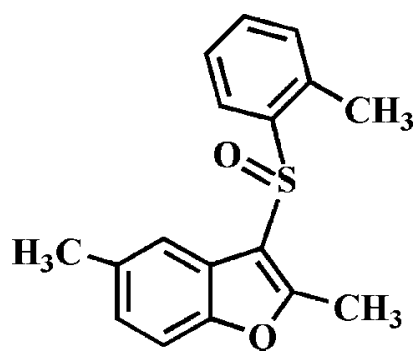

\section{Experimental}

2.1. Crystal data

$\mathrm{C}_{17} \mathrm{H}_{16} \mathrm{O}_{2} \mathrm{~S} \quad M_{r}=284.36$
Monoclinic, $P 2_{1} / n$

$a=10.8458(2) \AA$

$b=8.0139$ (1) $\AA$

$c=16.4295(2) \AA$

$\beta=96.709(1)^{\circ}$

$V=1418.23(4) \AA^{3}$

\subsection{Data collection}

Bruker SMART APEXII CCD diffractometer

Absorption correction: multi-scan (SADABS; Bruker, 2009)

$T_{\min }=0.692, T_{\max }=0.746$

\subsection{Refinement}

$R\left[F^{2}>2 \sigma\left(F^{2}\right)\right]=0.037$

$w R\left(F^{2}\right)=0.103$

$S=1.05$

3529 reflections
$Z=4$

Mo $K \alpha$ radiation

$\mu=0.23 \mathrm{~mm}^{-1}$

$T=173 \mathrm{~K}$

$0.44 \times 0.33 \times 0.30 \mathrm{~mm}$

Table 1

Hydrogen-bond geometry $\left(\AA,^{\circ}\right)$.

\begin{tabular}{lllll}
\hline$D-\mathrm{H} \cdots A$ & $D-\mathrm{H}$ & $\mathrm{H} \cdots A$ & $D \cdots A$ & $D-\mathrm{H} \cdots A$ \\
\hline $\mathrm{C} 6-\mathrm{H} 6 \cdots \mathrm{O} 2^{\mathrm{i}}$ & 0.95 & 2.45 & $3.3772(18)$ & 166 \\
$\mathrm{C} 17-\mathrm{H} 17 B \cdots \mathrm{O} 2^{\mathrm{ii}}$ & 0.98 & 2.55 & $3.458(2)$ & 154 \\
\hline
\end{tabular}

Symmetry codes: (i) $-x+\frac{1}{2}, y-\frac{1}{2},-z+\frac{3}{2}$; (ii) $x, y-1, z$.

Data collection: APEX2 (Bruker, 2009); cell refinement: SAINT (Bruker, 2009); data reduction: $S A I N T$; $\operatorname{program}(\mathrm{s})$ used to solve structure: SHELXS2014 (Sheldrick, 2008); program(s) used to refine structure: SHELXL2014 (Sheldrick, 2015); molecular graphics: ORTEP-3 for Windows (Farrugia, 2012) and DIAMOND (Brandenburg, 1998); software used to prepare material for publication: SHELXL2014 (Sheldrick, 2008).

\title{
Acknowledgements
}

This work was supported by a Dongeui University Grant (2015AA019). The X-ray centre of the Gyeongsang National University is acknowledged for providing access to the singlecrystal diffractometer.

Supporting information for this paper is available from the IUCr electronic archives (Reference: CV5492).

\section{References}

Akgul, Y. Y. \& Anil, H. (2003). Phytochemistry, 63, 939-943.

Aslam, S. N., Stevenson, P. C., Kokubun, T. \& Hall, D. R. (2009). Microbiol. Res. 164, 191-195.

Brandenburg, K. (1998). DIAMOND. Crystal Impact GbR, Bonn, Germany. Bruker (2009). APEX2, SADABS and SAINT. Bruker AXS Inc., Madison, Wisconsin, USA.

Choi, H. D., Seo, P. J. \& Lee, U. (2012). Acta Cryst. E68, o1410.

Choi, H. D., Seo, P. J. \& Son, B. W. (1999). J. Korean Chem. Soc. 43, 606-608. Farrugia, L. J. (2012). J. Appl. Cryst. 45, 849-854.

Galal, S. A., Abd El-All, A. S., Abdallah, M. M. \& El-Diwani, H. I. (2009). Bioorg. Med. Chem. Lett. 19, 2420-2428.

Howlett, D. R., Perry, A. E., Godfrey, F., Swatton, J. E., Jennings, K. H., Spitzfaden, C., Wadsworth, H., Wood, S. J. \& Markwell, R. E. (1999). Biochem. J. 340, 283-289. 


\section{data reports}

Ono, M., Kung, M. P., Hou, C. \& Kung, H. F. (2002). Nucl. Med. Biol. 29, 633642.

Sheldrick, G. M. (2008). Acta Cryst. A64, 112-122.

Sheldrick, G. M. (2015). Acta Cryst. C71, 3-8.
Soekamto, N. H., Achmad, S. A., Ghisalberti, E. L., Hakim, E. H. \& Syah, Y. M. (2003). Phytochemistry, 64, 831-834.

Wahab Khan, M., Jahangir Alam, M., Rashid, M. A. \& Chowdhury, R. (2005). Bioorg. Med. Chem. 13, 4796-4805. 


\section{supporting information}

Acta Cryst. (2015). E71, o554-o555 [https://doi.org/10.1107/S2056989015012773]

\section{Crystal structure of 2,5-dimethyl-3-(2-methylphenylsulfinyl)-1-benzofuran}

\section{Hong Dae Choi and Uk Lee}

\section{S1. Comment}

Benzofuran derivatives show interesting pharmacological properties such as antibacterial and antifungal, antitumor and antiviral, antimicrobial activities (Aslam et al. 2009; Galal et al., 2009; Wahab Khan et al., 2005), and potential inhibitor of $\beta$-amyloid aggregation (Howlett et al., 1999; Ono et al., 2002). These benzofuran compounds occur in a great number of natural products. (Akgul \& Anil, 2003; Soekamto et al., 2003). As a part of our continuing project on benzofuran derivatives (Choi et al., 2012), we report herein on the crystal structure of the title compound.

In the title molecule (Fig. 1), the benzofuran unit is essentially planar, with a mean deviation of 0.009 (1) $\AA$ from the least-squares plane defined by the nine constituent atoms. The 2-methylphenyl ring is essentially planar, with a mean deviation of 0.004 (1) $\AA$ from the least-squares plane defined by the six constituent atoms. The dihedral angle formed by the benzofuran ring and the 2-methylphenyl ring is $86.72(4)^{\circ}$. In the crystal, molecules are linked into a chain along the $b$ axis direction by $\mathrm{C}-\mathrm{H} \cdots \mathrm{O}$ hydrogen bonds (Table 1 and Fig. 2). These molecules are connected on either side of this chain by further $\mathrm{C}-\mathrm{H} \cdots \mathrm{O}$ hydrogen bonds (Table 1 and Fig. 2).

\section{S2. Experimental}

The starting material 2,5-dimethyl-3-(2-methylphenylsulfanyl)-1-benzofuran was prepared by literature method (Choi et al. 1999). 3-Chloroperoxybenzoic acid (77\%, $224 \mathrm{mg}, 1.0 \mathrm{mmol})$ was added in small portions to a stirred solution of 2,5dimethyl-3-(2-methylphenylsulfanyl)-1-benzofuran $(241 \mathrm{mg}, 0.9 \mathrm{mmol})$ in dichloromethane $(25 \mathrm{ml})$ at $273 \mathrm{~K}$. After being stirred at room temperature for $8 \mathrm{~h}$, the mixture was washed with saturated sodium bicarbonate solution ( $2 \mathrm{X} 10 \mathrm{ml})$ and the organic layer was separated, dried over magnesium sulfate, filtered and concentrated at reduced pressure. The residue was purified by column chromatography (hexane-ethyl acetate, $2: 1 \mathrm{v} / \mathrm{v}$ ) to afford the title compound as a colorless solid [yield 68\% (174 mg); m.p. 415-416 K; $R_{\mathrm{f}}=0.49$ (hexane-ethyl acetate, 2:1 v/v)]. Single crystals suitable for X-ray diffraction were prepared by slow evaporation of a solution of the title compound $(21 \mathrm{mg})$ in ethyl acetate $(20 \mathrm{ml})$ at room temperature.

\section{S3. Refinement}

All $\mathrm{H}$ atoms were positioned geometrically and refined using a riding model, with $\mathrm{C}-\mathrm{H}=0.95 \AA$ for aryl and $0.98 \AA$ for methyl $\mathrm{H}$ atoms, $U_{\text {iso }}(\mathrm{H})=1.2 U_{\text {eq }}(\mathrm{C})$ for aryl and $\left.1.5 U_{\text {eq }}(\mathrm{C})\right)$ for methyl $\mathrm{H}$ atoms. The positions of methyl hydrogens were optimized using the command AFIX in SHELXL-2014/7 (Sheldrick, 2015) 


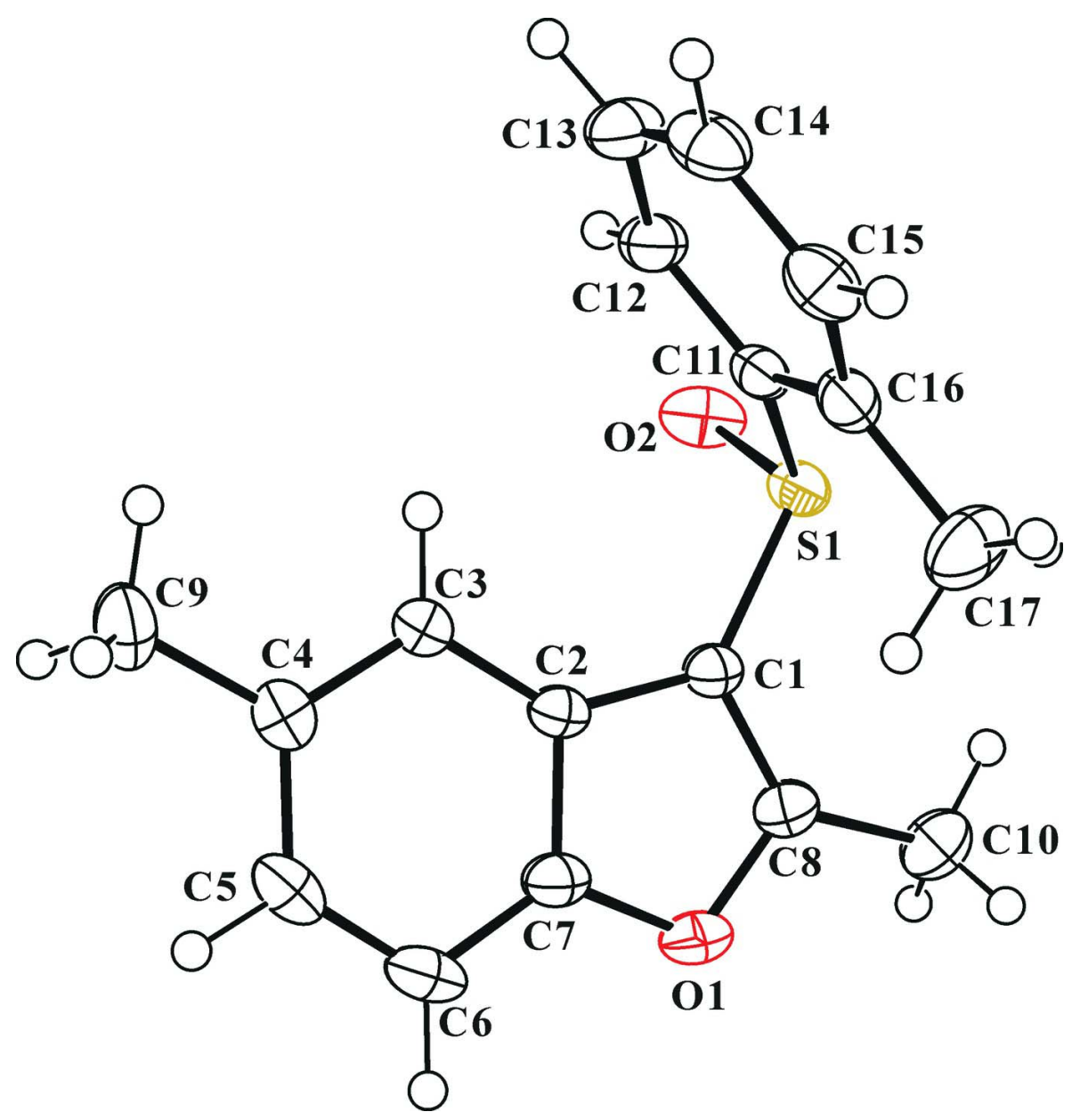

Figure 1

The molecular structure of the title compound, showing the atom-numbering scheme. Displacement ellipsoids are drawn at the $50 \%$ probability level. $\mathrm{H}$ atoms are presented as small spheres of arbitrary radius. 


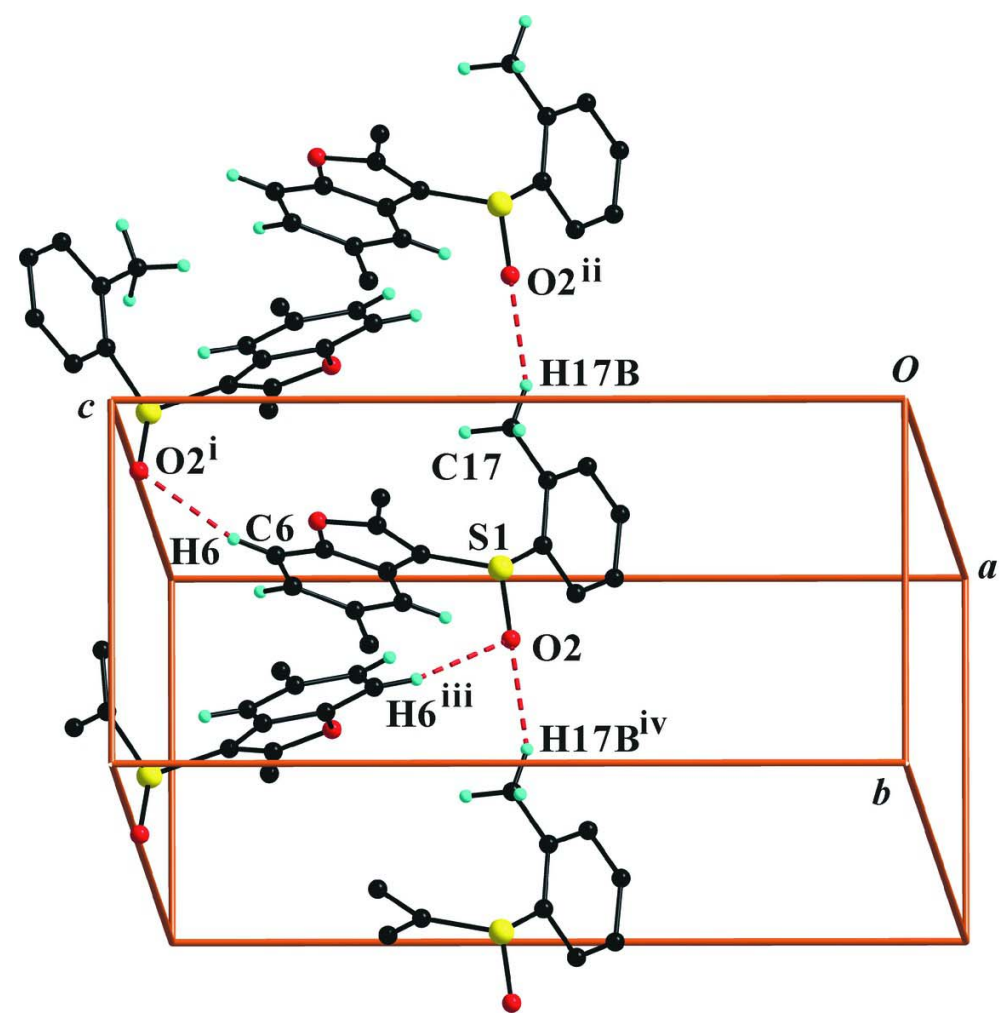

Figure 2

A view of the $\mathrm{C}-\mathrm{H} \cdots \mathrm{O}$ hydrogen bonds (dotted lines) in the crystal structure of the title compound. $\mathrm{H}$ atoms nonparticipating in hydrogen-bonding were omitted for clarity. [Symmetry codes: (i) $-x+1 / 2, y-1 / 2,-z+3 / 2$; (ii) $x, y-1$, $z$; (iii) $-x+1 / 2, y+1 / 2,-z+3 / 2$; (iv) $x, y+1, z$.]

\section{2,5-Dimethyl-3-(2-methylphenylsulfinyl)-1-benzofuran}

\section{Crystal data}

$\mathrm{C}_{17} \mathrm{H}_{16} \mathrm{O}_{2} \mathrm{~S}$

$M_{r}=284.36$

Monoclinic, $P 2_{1} / n$

$a=10.8458(2) \AA$

$b=8.0139$ (1) $\AA$

$c=16.4295$ (2) $\AA$

$\beta=96.709(1)^{\circ}$

$V=1418.23$ (4) $\AA^{3}$

$Z=4$

\section{Data collection}

\section{Bruker SMART APEXII CCD}

diffractometer

Radiation source: rotating anode

Graphite multilayer monochromator

Detector resolution: 10.0 pixels $\mathrm{mm}^{-1}$

$\varphi$ and $\omega$ scans

Absorption correction: multi-scan

(SADABS; Bruker, 2009)

$T_{\min }=0.692, T_{\max }=0.746$
$F(000)=600$

$D_{\mathrm{x}}=1.332 \mathrm{Mg} \mathrm{m}^{-3}$

Mo $K \alpha$ radiation, $\lambda=0.71073 \AA$

Cell parameters from 9125 reflections

$\theta=2.5-28.1^{\circ}$

$\mu=0.23 \mathrm{~mm}^{-1}$

$T=173 \mathrm{~K}$

Block, colourless

$0.44 \times 0.33 \times 0.30 \mathrm{~mm}$

25176 measured reflections

3529 independent reflections

3091 reflections with $I>2 \sigma(I)$

$R_{\text {int }}=0.033$

$\theta_{\text {max }}=28.4^{\circ}, \theta_{\min }=2.1^{\circ}$

$h=-14 \rightarrow 14$

$k=-10 \rightarrow 10$

$l=-21 \rightarrow 20$ 


\section{Refinement}

Refinement on $F^{2}$

Least-squares matrix: full

$R\left[F^{2}>2 \sigma\left(F^{2}\right)\right]=0.037$

$w R\left(F^{2}\right)=0.103$

$S=1.05$

3529 reflections

184 parameters

0 restraints

Primary atom site location: structure-invariant direct methods
Secondary atom site location: difference Fourier map

Hydrogen site location: difference Fourier map

$\mathrm{H}$-atom parameters constrained

$w=1 /\left[\sigma^{2}\left(F_{\mathrm{o}}{ }^{2}\right)+(0.0515 P)^{2}+0.5244 P\right]$

where $P=\left(F_{\mathrm{o}}^{2}+2 F_{\mathrm{c}}^{2}\right) / 3$

$(\Delta / \sigma)_{\max }=0.001$

$\Delta \rho_{\max }=0.30 \mathrm{e} \AA^{-3}$

$\Delta \rho_{\min }=-0.27{\mathrm{e} \AA^{-3}}^{-3}$

Special details

Experimental. ${ }^{1} \mathrm{H}$ NMR $\left(\delta\right.$ p.p.m., $\left.\mathrm{CDCl}_{3}, 400 \mathrm{~Hz}\right): 8.35(d, J=7.88 \mathrm{~Hz}, 1 \mathrm{H}), 7.53-7.57(\mathrm{~m}, 1 \mathrm{H}), 7.38-7.42(\mathrm{~m}, 1 \mathrm{H})$, $7.26(d, J=7.02 \mathrm{~Hz}, 1 \mathrm{H}), 7.15(d, J=7.52 \mathrm{~Hz}, 1 \mathrm{H}), 6.98-7.03(\mathrm{~m}, 2 \mathrm{H}), 2.73(\mathrm{~s}, 3 \mathrm{H}), 2.25(\mathrm{~s}, 3 \mathrm{H}), 2.13(\mathrm{~s}, 3 \mathrm{H})$,

Geometry. All esds (except the esd in the dihedral angle between two 1.s. planes) are estimated using the full covariance matrix. The cell esds are taken into account individually in the estimation of esds in distances, angles and torsion angles; correlations between esds in cell parameters are only used when they are defined by crystal symmetry. An approximate (isotropic) treatment of cell esds is used for estimating esds involving l.s. planes.

Fractional atomic coordinates and isotropic or equivalent isotropic displacement parameters $\left(\AA^{2}\right)$

\begin{tabular}{lllll}
\hline & $x$ & $y$ & $z$ & $U_{\text {iso }} * / U_{\text {eq }}$ \\
\hline S1 & $0.17973(3)$ & $0.37549(4)$ & $0.52324(2)$ & $0.02675(11)$ \\
O1 & $0.17654(10)$ & $0.24730(14)$ & $0.75315(6)$ & $0.0349(2)$ \\
O2 & $0.21361(11)$ & $0.55407(13)$ & $0.51308(6)$ & $0.0381(3)$ \\
C1 & $0.21664(12)$ & $0.31930(17)$ & $0.62666(8)$ & $0.0250(3)$ \\
C2 & $0.33439(12)$ & $0.30795(16)$ & $0.67783(8)$ & $0.0236(3)$ \\
C3 & $0.45906(12)$ & $0.33131(16)$ & $0.66789(8)$ & $0.0255(3)$ \\
H3 & 0.4825 & 0.3619 & 0.6160 & $0.031^{*}$ \\
C4 & $0.54881(13)$ & $0.30933(18)$ & $0.73475(9)$ & $0.0307(3)$ \\
C5 & $0.51194(15)$ & $0.2614(2)$ & $0.81059(9)$ & $0.0363(3)$ \\
H5 & 0.5740 & 0.2456 & 0.8557 & $0.044^{*}$ \\
C6 & $0.38937(16)$ & $0.23627(19)$ & $0.82225(9)$ & $0.0355(3)$ \\
H6 & 0.3656 & 0.2031 & 0.8737 & $0.043^{*}$ \\
C7 & $0.30345(13)$ & $0.26225(18)$ & $0.75471(8)$ & $0.0287(3)$ \\
C8 & $0.12638(13)$ & $0.28335(19)$ & $0.67489(9)$ & $0.0304(3)$ \\
C9 & $0.68418(15)$ & $0.3366(2)$ & $0.72713(11)$ & $0.0434(4)$ \\
H9A & 0.6941 & 0.3692 & 0.6708 & $0.065^{*}$ \\
H9B & 0.7301 & 0.2331 & 0.7408 & $0.065^{*}$ \\
H9C & 0.7165 & 0.4252 & 0.7648 & $0.065^{*}$ \\
C10 & $-0.01086(15)$ & $0.2761(3)$ & $0.65979(11)$ & $0.0452(4)$ \\
H10A & -0.0380 & 0.3087 & 0.6030 & $0.068^{*}$ \\
H10B & -0.0466 & 0.3527 & 0.6972 & $0.068^{*}$ \\
H10C & -0.0388 & 0.1622 & 0.6691 & $0.068^{*}$ \\
C11 & $0.29473(12)$ & $0.25559(17)$ & $0.47850(7)$ & $0.0236(3)$ \\
C12 & $0.38520(14)$ & $0.34425(18)$ & $0.44408(8)$ & $0.0297(3)$ \\
H12 & 0.3893 & 0.4622 & 0.4493 & $0.036^{*}$ \\
C13 & $0.46993(15)$ & $0.2599(2)$ & $0.40181(9)$ & $0.0365(3)$ \\
H13 & 0.5331 & 0.3197 & 0.3788 & $0.044^{*}$ \\
& & & &
\end{tabular}




\begin{tabular}{lllll} 
C14 & $0.46160(15)$ & $0.0895(2)$ & $0.39362(9)$ & $0.0363(3)$ \\
H14 & 0.5183 & 0.0315 & 0.3639 & $0.044^{*}$ \\
C15 & $0.37125(14)$ & $0.00203(19)$ & $0.42834(9)$ & $0.0338(3)$ \\
H15 & 0.3675 & -0.1159 & 0.4225 & $0.041^{*}$ \\
C16 & $0.28551(13)$ & $0.08234(18)$ & $0.47168(8)$ & $0.0285(3)$ \\
C17 & $0.18974(17)$ & $-0.0156(2)$ & $0.50997(12)$ & $0.0464(4)$ \\
H17A & 0.2083 & -0.0119 & 0.5698 & $0.070^{*}$ \\
H17B & 0.1907 & -0.1318 & 0.4914 & $0.070^{*}$ \\
H17C & 0.1075 & 0.0326 & 0.4938 & $0.070^{*}$ \\
\hline
\end{tabular}

Atomic displacement parameters $\left(\AA^{2}\right)$

\begin{tabular}{lllllll}
\hline & $U^{11}$ & $U^{22}$ & $U^{33}$ & $U^{12}$ & $U^{13}$ & $U^{23}$ \\
\hline S1 & $0.02608(18)$ & $0.03273(19)$ & $0.02139(17)$ & $0.00435(12)$ & $0.00261(12)$ & $0.00056(12)$ \\
O1 & $0.0357(6)$ & $0.0446(6)$ & $0.0266(5)$ & $-0.0031(5)$ & $0.0128(4)$ & $0.0039(4)$ \\
O2 & $0.0555(7)$ & $0.0288(5)$ & $0.0317(5)$ & $0.0098(5)$ & $0.0130(5)$ & $0.0029(4)$ \\
C1 & $0.0253(6)$ & $0.0285(6)$ & $0.0219(6)$ & $0.0001(5)$ & $0.0053(5)$ & $0.0000(5)$ \\
C2 & $0.0290(6)$ & $0.0229(6)$ & $0.0194(6)$ & $0.0002(5)$ & $0.0046(5)$ & $-0.0003(4)$ \\
C3 & $0.0266(6)$ & $0.0280(6)$ & $0.0220(6)$ & $-0.0007(5)$ & $0.0026(5)$ & $-0.0005(5)$ \\
C4 & $0.0314(7)$ & $0.0304(7)$ & $0.0292(7)$ & $0.0008(5)$ & $-0.0014(6)$ & $-0.0019(5)$ \\
C5 & $0.0433(8)$ & $0.0393(8)$ & $0.0238(7)$ & $0.0031(6)$ & $-0.0064(6)$ & $0.0009(6)$ \\
C6 & $0.0515(9)$ & $0.0353(8)$ & $0.0198(6)$ & $0.0018(7)$ & $0.0052(6)$ & $0.0032(5)$ \\
C7 & $0.0331(7)$ & $0.0312(7)$ & $0.0230(6)$ & $-0.0009(5)$ & $0.0084(5)$ & $0.0008(5)$ \\
C8 & $0.0289(7)$ & $0.0362(7)$ & $0.0275(7)$ & $-0.0020(5)$ & $0.0089(5)$ & $-0.0005(5)$ \\
C9 & $0.0293(8)$ & $0.0558(10)$ & $0.0424(9)$ & $-0.0019(7)$ & $-0.0066(7)$ & $-0.0019(7)$ \\
C10 & $0.0281(8)$ & $0.0646(11)$ & $0.0451(9)$ & $-0.0056(7)$ & $0.0132(7)$ & $-0.0002(8)$ \\
C11 & $0.0245(6)$ & $0.0286(7)$ & $0.0171(5)$ & $0.0019(5)$ & $0.0003(5)$ & $-0.0007(4)$ \\
C12 & $0.0344(7)$ & $0.0309(7)$ & $0.0249(6)$ & $-0.0023(5)$ & $0.0073(5)$ & $-0.0004(5)$ \\
C13 & $0.0363(8)$ & $0.0450(9)$ & $0.0304(7)$ & $0.0001(6)$ & $0.0128(6)$ & $0.0006(6)$ \\
C14 & $0.0373(8)$ & $0.0457(9)$ & $0.0263(7)$ & $0.0124(7)$ & $0.0049(6)$ & $-0.0039(6)$ \\
C15 & $0.0418(8)$ & $0.0299(7)$ & $0.0285(7)$ & $0.0069(6)$ & $-0.0015(6)$ & $-0.0039(5)$ \\
C16 & $0.0313(7)$ & $0.0300(7)$ & $0.0232(6)$ & $-0.0014(5)$ & $-0.0011(5)$ & $-0.0005(5)$ \\
C17 & $0.0526(10)$ & $0.0321(8)$ & $0.0570(11)$ & $-0.0126(7)$ & $0.0168(8)$ & $-0.0016(7)$ \\
& & & & & & \\
\hline
\end{tabular}

Geometric parameters $\left(\AA,{ }^{\circ}\right)$

\begin{tabular}{llll}
\hline $\mathrm{S} 1-\mathrm{O} 2$ & $1.4918(11)$ & $\mathrm{C} 9-\mathrm{H} 9 \mathrm{~B}$ & 0.9800 \\
$\mathrm{~S} 1-\mathrm{C} 1$ & $1.7580(13)$ & $\mathrm{C} 9-\mathrm{H} 9 \mathrm{C}$ & 0.9800 \\
$\mathrm{~S} 1-\mathrm{C} 11$ & $1.7982(13)$ & $\mathrm{C} 10-\mathrm{H} 10 \mathrm{~A}$ & 0.9800 \\
$\mathrm{O} 1-\mathrm{C} 8$ & $1.3671(18)$ & $\mathrm{C} 10-\mathrm{H} 10 \mathrm{~B}$ & 0.9800 \\
$\mathrm{O} 1-\mathrm{C} 7$ & $1.3789(17)$ & $\mathrm{C} 10-\mathrm{H} 10 \mathrm{C}$ & 0.9800 \\
$\mathrm{C} 1-\mathrm{C} 8$ & $1.3604(18)$ & $\mathrm{C} 11-\mathrm{C} 12$ & $1.3846(19)$ \\
$\mathrm{C} 1-\mathrm{C} 2$ & $1.4476(18)$ & $\mathrm{C} 11-\mathrm{C} 16$ & $1.3955(19)$ \\
$\mathrm{C} 2-\mathrm{C} 7$ & $1.3932(17)$ & $\mathrm{C} 12-\mathrm{C} 13$ & $1.390(2)$ \\
$\mathrm{C} 2-\mathrm{C} 3$ & $1.3934(18)$ & $\mathrm{C} 12-\mathrm{H} 12$ & 0.9500 \\
$\mathrm{C} 3-\mathrm{C} 4$ & $1.3912(19)$ & $\mathrm{C} 13-\mathrm{C} 14$ & $1.375(2)$ \\
$\mathrm{C} 3-\mathrm{H} 3$ & 0.9500 & $\mathrm{C} 13-\mathrm{H} 13$ & 0.9500 \\
$\mathrm{C} 4-\mathrm{C} 5$ & $1.406(2)$ & $\mathrm{C} 14-\mathrm{C} 15$ & $1.381(2)$
\end{tabular}




\begin{tabular}{|c|c|c|c|}
\hline $\mathrm{C} 4-\mathrm{C} 9$ & $1.504(2)$ & $\mathrm{C} 14-\mathrm{H} 14$ & 0.9500 \\
\hline $\mathrm{C} 5-\mathrm{C} 6$ & $1.380(2)$ & $\mathrm{C} 15-\mathrm{C} 16$ & $1.393(2)$ \\
\hline $\mathrm{C} 5-\mathrm{H} 5$ & 0.9500 & $\mathrm{C} 15-\mathrm{H} 15$ & 0.9500 \\
\hline $\mathrm{C} 6-\mathrm{C} 7$ & $1.379(2)$ & $\mathrm{C} 16-\mathrm{C} 17$ & $1.498(2)$ \\
\hline $\mathrm{C} 6-\mathrm{H} 6$ & 0.9500 & $\mathrm{C} 17-\mathrm{H} 17 \mathrm{~A}$ & 0.9800 \\
\hline $\mathrm{C} 8-\mathrm{C} 10$ & $1.481(2)$ & C17-H17B & 0.9800 \\
\hline C9-H9A & 0.9800 & $\mathrm{C} 17-\mathrm{H} 17 \mathrm{C}$ & 0.9800 \\
\hline $\mathrm{O} 2-\mathrm{S} 1-\mathrm{C} 1$ & $108.82(6)$ & $\mathrm{H} 9 \mathrm{~A}-\mathrm{C} 9-\mathrm{H} 9 \mathrm{C}$ & 109.5 \\
\hline $\mathrm{O} 2-\mathrm{S} 1-\mathrm{C} 11$ & $105.97(6)$ & $\mathrm{H} 9 \mathrm{~B}-\mathrm{C} 9-\mathrm{H} 9 \mathrm{C}$ & 109.5 \\
\hline $\mathrm{C} 1-\mathrm{S} 1-\mathrm{C} 11$ & $99.66(6)$ & $\mathrm{C} 8-\mathrm{C} 10-\mathrm{H} 10 \mathrm{~A}$ & 109.5 \\
\hline $\mathrm{C} 8-\mathrm{O} 1-\mathrm{C} 7$ & $106.61(10)$ & $\mathrm{C} 8-\mathrm{C} 10-\mathrm{H} 10 \mathrm{~B}$ & 109.5 \\
\hline $\mathrm{C} 8-\mathrm{C} 1-\mathrm{C} 2$ & $107.12(12)$ & $\mathrm{H} 10 \mathrm{~A}-\mathrm{C} 10-\mathrm{H} 10 \mathrm{~B}$ & 109.5 \\
\hline $\mathrm{C} 8-\mathrm{C} 1-\mathrm{S} 1$ & $121.27(11)$ & $\mathrm{C} 8-\mathrm{C} 10-\mathrm{H} 10 \mathrm{C}$ & 109.5 \\
\hline $\mathrm{C} 2-\mathrm{C} 1-\mathrm{S} 1$ & $131.55(10)$ & $\mathrm{H} 10 \mathrm{~A}-\mathrm{C} 10-\mathrm{H} 10 \mathrm{C}$ & 109.5 \\
\hline $\mathrm{C} 7-\mathrm{C} 2-\mathrm{C} 3$ & $118.78(12)$ & $\mathrm{H} 10 \mathrm{~B}-\mathrm{C} 10-\mathrm{H} 10 \mathrm{C}$ & 109.5 \\
\hline $\mathrm{C} 7-\mathrm{C} 2-\mathrm{C} 1$ & $104.68(11)$ & $\mathrm{C} 12-\mathrm{C} 11-\mathrm{C} 16$ & $121.69(12)$ \\
\hline $\mathrm{C} 3-\mathrm{C} 2-\mathrm{C} 1$ & $136.54(12)$ & $\mathrm{C} 12-\mathrm{C} 11-\mathrm{S} 1$ & $116.82(10)$ \\
\hline $\mathrm{C} 4-\mathrm{C} 3-\mathrm{C} 2$ & $119.30(13)$ & $\mathrm{C} 16-\mathrm{C} 11-\mathrm{S} 1$ & $121.19(10)$ \\
\hline $\mathrm{C} 4-\mathrm{C} 3-\mathrm{H} 3$ & 120.3 & $\mathrm{C} 11-\mathrm{C} 12-\mathrm{C} 13$ & $119.74(14)$ \\
\hline $\mathrm{C} 2-\mathrm{C} 3-\mathrm{H} 3$ & 120.3 & $\mathrm{C} 11-\mathrm{C} 12-\mathrm{H} 12$ & 120.1 \\
\hline $\mathrm{C} 3-\mathrm{C} 4-\mathrm{C} 5$ & $119.29(13)$ & $\mathrm{C} 13-\mathrm{C} 12-\mathrm{H} 12$ & 120.1 \\
\hline $\mathrm{C} 3-\mathrm{C} 4-\mathrm{C} 9$ & $121.04(14)$ & $\mathrm{C} 14-\mathrm{C} 13-\mathrm{C} 12$ & $119.48(14)$ \\
\hline $\mathrm{C} 5-\mathrm{C} 4-\mathrm{C} 9$ & $119.67(14)$ & $\mathrm{C} 14-\mathrm{C} 13-\mathrm{H} 13$ & 120.3 \\
\hline $\mathrm{C} 6-\mathrm{C} 5-\mathrm{C} 4$ & $122.77(13)$ & $\mathrm{C} 12-\mathrm{C} 13-\mathrm{H} 13$ & 120.3 \\
\hline $\mathrm{C} 6-\mathrm{C} 5-\mathrm{H} 5$ & 118.6 & $\mathrm{C} 13-\mathrm{C} 14-\mathrm{C} 15$ & $120.36(14)$ \\
\hline $\mathrm{C} 4-\mathrm{C} 5-\mathrm{H} 5$ & 118.6 & $\mathrm{C} 13-\mathrm{C} 14-\mathrm{H} 14$ & 119.8 \\
\hline $\mathrm{C} 7-\mathrm{C} 6-\mathrm{C} 5$ & $115.96(13)$ & $\mathrm{C} 15-\mathrm{C} 14-\mathrm{H} 14$ & 119.8 \\
\hline $\mathrm{C} 7-\mathrm{C} 6-\mathrm{H} 6$ & 122.0 & $\mathrm{C} 14-\mathrm{C} 15-\mathrm{C} 16$ & $121.67(14)$ \\
\hline $\mathrm{C} 5-\mathrm{C} 6-\mathrm{H} 6$ & 122.0 & $\mathrm{C} 14-\mathrm{C} 15-\mathrm{H} 15$ & 119.2 \\
\hline $\mathrm{O} 1-\mathrm{C} 7-\mathrm{C} 6$ & $125.53(13)$ & $\mathrm{C} 16-\mathrm{C} 15-\mathrm{H} 15$ & 119.2 \\
\hline $\mathrm{O} 1-\mathrm{C} 7-\mathrm{C} 2$ & $110.59(12)$ & $\mathrm{C} 15-\mathrm{C} 16-\mathrm{C} 11$ & $117.05(13)$ \\
\hline $\mathrm{C} 6-\mathrm{C} 7-\mathrm{C} 2$ & $123.88(14)$ & $\mathrm{C} 15-\mathrm{C} 16-\mathrm{C} 17$ & 120.63 \\
\hline $\mathrm{C} 1-\mathrm{C} 8-\mathrm{O} 1$ & $110.99(12)$ & $\mathrm{C} 11-\mathrm{C} 16-\mathrm{C} 17$ & $122.31(13)$ \\
\hline $\mathrm{C} 1-\mathrm{C} 8-\mathrm{C} 10$ & $133.48(14)$ & $\mathrm{C} 16-\mathrm{C} 17-\mathrm{H} 17 \mathrm{~A}$ & 109.5 \\
\hline $\mathrm{O} 1-\mathrm{C} 8-\mathrm{C} 10$ & $115.53(12)$ & $\mathrm{C} 16-\mathrm{C} 17-\mathrm{H} 17 \mathrm{~B}$ & 109.5 \\
\hline $\mathrm{C} 4-\mathrm{C} 9-\mathrm{H} 9 \mathrm{~A}$ & 109.5 & $\mathrm{H} 17 \mathrm{~A}-\mathrm{C} 17-\mathrm{H} 17 \mathrm{~B}$ & 109.5 \\
\hline $\mathrm{C} 4-\mathrm{C} 9-\mathrm{H} 9 \mathrm{~B}$ & 109.5 & $\mathrm{C} 16-\mathrm{C} 17-\mathrm{H} 17 \mathrm{C}$ & 109.5 \\
\hline $\mathrm{H} 9 \mathrm{~A}-\mathrm{C} 9-\mathrm{H} 9 \mathrm{~B}$ & 109.5 & $\mathrm{H} 17 \mathrm{~A}-\mathrm{C} 17-\mathrm{H} 17 \mathrm{C}$ & 109.5 \\
\hline $\mathrm{C} 4-\mathrm{C} 9-\mathrm{H} 9 \mathrm{C}$ & 109.5 & $\mathrm{H} 17 \mathrm{~B}-\mathrm{C} 17-\mathrm{H} 17 \mathrm{C}$ & 109.5 \\
\hline
\end{tabular}

Hydrogen-bond geometry $\left(A,{ }^{\circ}\right)$

\begin{tabular}{lllll}
\hline$D-\mathrm{H} \cdots A$ & $D-\mathrm{H}$ & $\mathrm{H} \cdots A$ & $D \cdots A$ & $D-\mathrm{H} \cdots A$ \\
\hline $\mathrm{C} 6-\mathrm{H} 6 \cdots \mathrm{O} 2^{\mathrm{i}}$ & 0.95 & 2.45 & $3.3772(18)$ & 166 \\
$\mathrm{C} 17-\mathrm{H} 17 B^{\cdots} \cdots 2^{\mathrm{ii}}$ & 0.98 & 2.55 & $3.458(2)$ & 154 \\
\hline
\end{tabular}

Symmetry codes: (i) $-x+1 / 2, y-1 / 2,-z+3 / 2$; (ii) $x, y-1, z$. 